\title{
In reply: Ultrasound-guided internal jugular vein cannulation: techniques to confirm guidewire location
}

\author{
Kazuyoshi Aoyama, MD • Ichiro Takenaka, MD
}

Received: 11 August 2015/Accepted: 17 August 2015/Published online: 26 August 2015

(C) Canadian Anesthesiologists' Society 2015

\section{To the Editor,}

We thank Drs Maheshwari for their interest in our report $^{1}$ in which we described the application of digital neck pressure to facilitate visualization of the guidewire within the venous lumen during ultrasound-guided internal jugular vein cannulation. They propose three additional maneuvers for confirmation of the guidewire within the vein: placing the ultrasound probe in plane, wiggling the guidewire, and applying the pressure with the ultrasound transducer and its release. Our method, as are theirs, is simple, noninvasive and quickly performed.

The guidewire is not always visible with the ultrasound image, ${ }^{2}$ and we entirely agree that multiple techniques should be used to confirm correct placement. In our view, confirmation of the guidewire is also important for patient safety, although there is little information about the specifics of ultrasound-guided guidewire visualization in most reported studies.

Conflicts of interest None declared.

Funding Support was provided solely from institutional and/or departmental sources.

\section{References}

1. Aoyama K, Takenaka I, Iwagaki T, Sano H. A simple maneuver for confirmation of the guidewire during ultrasound-guided internal jugular vein cannulation. Can J Anesth 2015; 62: 839-40.

2. Moak JH, Lyons MS, Wright SW, Lindsell CJ. Needle and guidewire visualization in ultrasound-guided internal jugular vein cannulation. Am J Emerg Med 2011; 29: 432-6.

K. Aoyama, MD ( $\square)$

Department of Anesthesia, Kitakyushu General Hospital,

Kitakyushu, Japan

e-mail: kazuyoshi.aoyama@kitakyu-hp.or.jp

I. Takenaka, MD

Department of Anesthesia, Kyushu Rosai Hospital, Kitakyushu,

Japan 